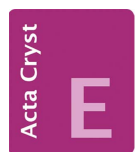

CRYSTALLOGRAPHIC COMMUNICATIONS

ISSN 2056-9890

Received 17 February 2021

Accepted 21 May 2021

Edited by A. V. Yatsenko, Moscow State University, Russia

Keywords: crystal structure; diketone; $\mathrm{C}-\mathrm{H} \cdots \mathrm{F}$ interactions.

CCDC reference: 2085161

Supporting information: this article has supporting information at journals.iucr.org/e

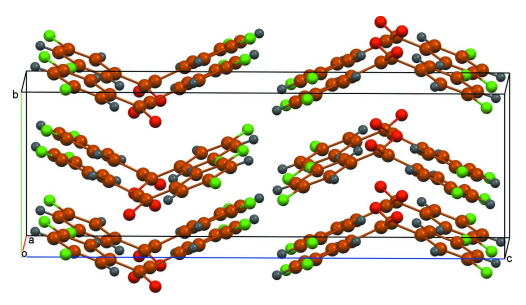

open $\odot$ ACCESS

\section{Crystal structure of 1,2-bis(3,5-difluorophenyl)- ethane-1,2-dione}

\author{
Loren C. Brown* and Gary J. Balaich \\ Department of Chemistry \& Chemistry Research Center, United States Air Force Academy, Colorado Springs, CO 80840, \\ USA. *Correspondence e-mail: Gary.Balaich@usafa.edu
}

The title compound, $\mathrm{C}_{14} \mathrm{H}_{6} \mathrm{~F}_{4} \mathrm{O}_{2}$, crystallizes with half of a molecule per asymmetric unit and exhibits bond lengths and angles typical of $\alpha$-diketones. A network of $\mathrm{C}-\mathrm{H} \cdots \mathrm{F}$ contacts and $\pi-\pi$ stacking interactions is observed within the structure.

\section{Chemical context}

Aryl diketones are a class of dicarbonyl compounds with a wide variety of uses in organic synthesis. The title $\alpha$-diketone, 1,2-bis(3,5-difluorophenyl)ethane-1,2-dione, is used as a precursor in the production of hexabenzocoronenes (Jones et al., 2012). More recently, 1,2-bis(3,5-difluorophenyl)ethane1,2-dione has been used in the synthesis of various polymers that have been studied for photovoltaics (Cai et al., 2019) and for gas chromatography (GC) stationary phases (Liu et al., 2019). Although the synthetic chemistry is known in the literature, to the best of our knowledge, structural data have not yet been published for the title compound. Herein we report the crystal structure of 1,2-bis(3,5-difluorophenyl)ethane-1,2-dione, isolated as a minor impurity in the synthesis of the related 1,4-diarylketone, 1-(3,5-difluorophenyl)pentane-1,4-dione.<smiles>O=C(C(=O)c1cc(F)cc(F)c1)c1cc(F)cc(F)c1</smiles>

\section{Structural commentary}

The title compound (Fig. 1) crystallized in the orthorhombic space group Pbcn. Benzil (1,2-diphenylethane-1,2-dione) and similar $\alpha, \alpha$-diketones crystallize in trigonal or monoclinic space groups, respectively (Charpe et al., 2020; El Moncef $e t$ al., 2010; Fun et al., 2008). The title compound crystallizes with one half-molecule per asymmetric unit $\left(Z^{\prime}=0.5\right)$, and exhibits the expected bond lengths and angles for $\alpha$-diketone $s p^{2}$ hybridized atoms. Interestingly, the $\mathrm{C} 5-\mathrm{C} 6-\mathrm{C} 7-\mathrm{O} 1$ torsion angle $\left[7.55(19)^{\circ}\right]$ of the title compound is larger compared to 


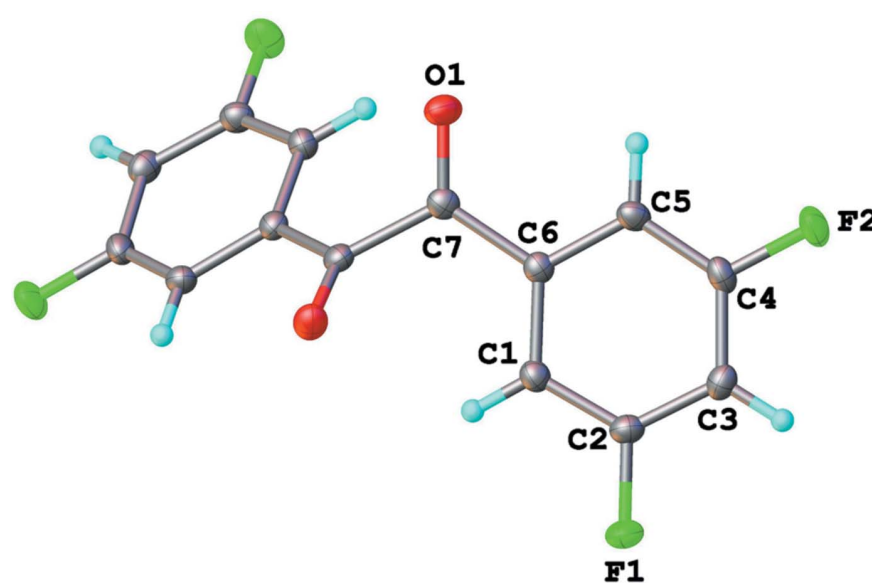

Figure 1

The molecular structure of 1,2-bis(3,5-difluorophenyl)ethane-1,2-dione. Displacement ellipsoids are shown at the $50 \%$ probability level.

the same torsion angle in bis(4-fluorophenyl)ethane-1,2-dione [5.69 (18) ${ }^{\circ}$; Fun et al., 2008] and benzil [3.80 (18) ${ }^{\circ}$; Charpe et al., 2020], but smaller compared to 1,2-bis(3-methoxyphen-

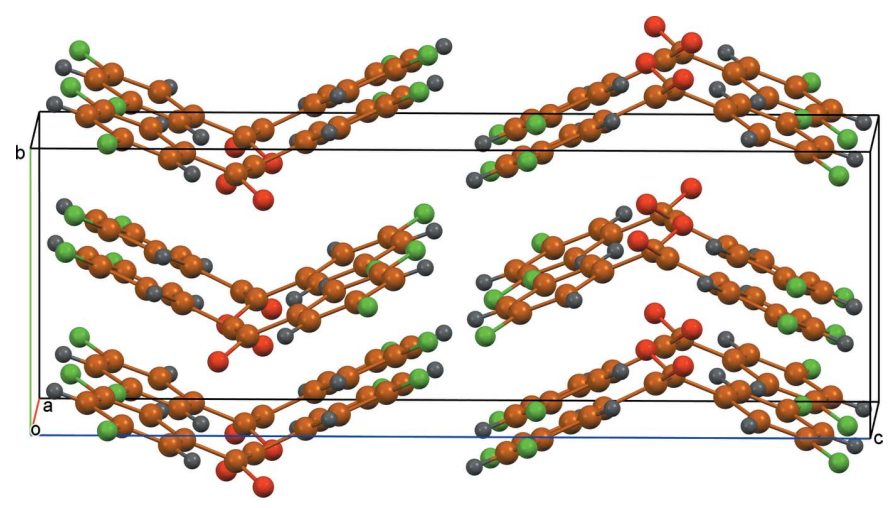

Figure 2

A packing view of 1,2-bis(3,5-difluorophenyl)ethane-1,2-dione.

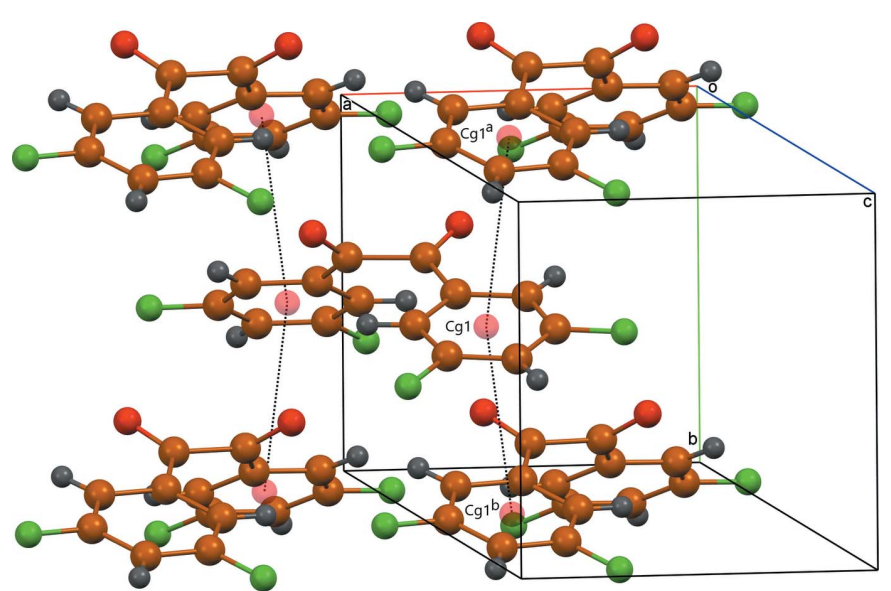

Figure 3

View of $\pi-\pi$ stacking interactions in the title structure. Short intercentroid separations are shown by dashed lines. $C g 1$ is the centroid of the C1-C6 ring.Symmetry codes: $(a) \frac{3}{2}-x,-\frac{1}{2}+y, z ;(b) \frac{3}{2}-x, \frac{1}{2}+y, z$.
Table 1

Hydrogen-bond geometry $\left(\AA{ }^{\circ}\right)$.

\begin{tabular}{lllll}
\hline$D-\mathrm{H} \cdots A$ & $D-\mathrm{H}$ & $\mathrm{H} \cdots A$ & $D \cdots A$ & $D-\mathrm{H} \cdots A$ \\
\hline $\mathrm{C} 1-\mathrm{H} 1 \cdots \mathrm{F}^{\mathrm{i}}$ & 0.93 & 2.48 & $3.2281(16)$ & 137 \\
$\mathrm{C} 3-\mathrm{H} 3 \cdots 1^{\mathrm{ii}}$ & 0.93 & 2.46 & $3.3211(15)$ & 154 \\
$\mathrm{C} 5-\mathrm{H} 5 \cdots 1^{\mathrm{iii}}$ & 0.93 & 2.65 & $3.517(2)$ & 156 \\
\hline
\end{tabular}

Symmetry codes: (i) $x+1, y, z$; (ii) $x-\frac{1}{2},-y+\frac{3}{2},-z+1$; (iii) $-x+1, y,-z+\frac{1}{2}$.

yl)ethane-1,2-dione [7.94 $(15)^{\circ}$; Goossens et al., 2005]. The dihedral angle between the two rings is $49.50(6)^{\circ}$ with a C6$\mathrm{C} 7-\mathrm{C}^{\prime}$ - $\mathrm{C}^{\prime}$ 'torsion angle of $125.92(5)^{\circ}$.

\section{Supramolecular features}

A view of crystal packing of the title compound is presented in Fig. 2. The molecules pack in a stacking pattern maximizing slipped $\pi-\pi$ stacking interactions between planes of the difluoroaryl rings with an intercentroid separation of 3.7317 (8) $\AA$, thus forming layers parallel to the $b c$ plane (Fig. 3). Similar $\pi-\pi$ stacking interactions with comparable intercentroid separations were observed in bis(4-fluorophenyl)ethane-1,2-dione [3.6416 (9) Аं; Fun et al., 2008] and benzil [3.7566 (17) А; Charpe et al., 2020]. As a result of the packing arrangement of bis(3-methoxyphenyl)ethane-1,2-dione, no $\pi$ $\pi$ stacking interactions were observed (Goossens et al., 2005). The title compound packs in a way that allows close contacts between the fluorine atoms and hydrogen atoms of adjacent molecules, leading to a network of $\mathrm{C}-\mathrm{H} \cdots \mathrm{F}$ interactions (Table 1, Fig. 4) as well as fluorine interactions between neighboring molecules $[\mathrm{F} 1 \cdots \mathrm{F} 2(1+x, y, z)=2.9372$ (16) $\AA$, $\mathrm{F} 1 \cdots \mathrm{F} 1(2-x, 1-y, 1-z)=2.8614(16) \AA]$. A network of $\mathrm{C}-\mathrm{H} \cdots \mathrm{O}$ interactions is also observed between the carbonyl oxygen and H5. This interaction is significantly weaker for 1,2bis(3,5-difluorophenyl)ethane-1,2-dione in comparison to benzil $(\mathrm{O} \cdots \mathrm{H}=2.42 \AA)$ and bis(4-fluorophenyl)ethane-1,2dione $(\mathrm{O} \cdots \mathrm{H}=2.40 \AA$ ). As a result, the $\pi-\pi$ stacking and $\mathrm{C}-$ $\mathrm{H} \cdots \mathrm{F}$ interactions play a vital role in how the compound packs within the crystal structure.

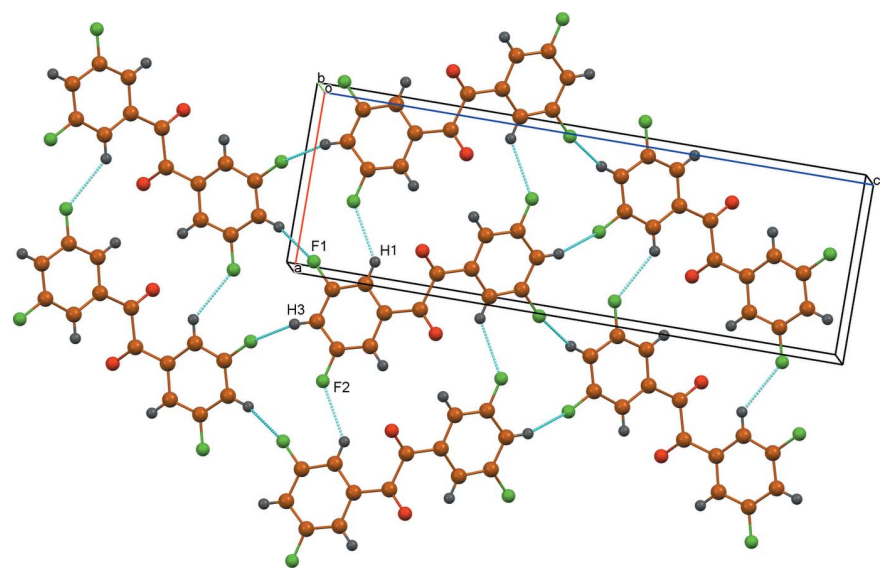

Figure 4

View of intermolecular $\mathrm{C}-\mathrm{H} \cdots \mathrm{F}$ interactions in the title structure (for details see Table 1$)$. 


\section{Database survey}

A search of the Cambridge Structural Database (CSD, version of December 2019; Groom et al., 2016) for aryl substituted $\alpha$-diketones yielded 178 results. The bond lengths and angles in the title molecule are consistent with $\alpha, \alpha$-diketones reported in the literature. The most closely related compound was compared to the title compound in the preceeding sections.

\section{Synthesis and crystallization}

Colorless crystals of the title compound suitable for singlecrystal X-ray diffraction study were obtained by slow evaporation of a dichloromethane solution of the residue left after isolation of 1-(3,5-difluorophenyl)pentane-1,4-dione.

\section{Refinement}

Crystal data, data collection and structure refinement details are summarized in Table 2. All $\mathrm{H}$ atoms were positioned geometrically $(\mathrm{C}-\mathrm{H}=0.93 \AA)$ and refined as riding with $U_{\text {iso }}(\mathrm{H})=1.2 U_{\text {eq }}(\mathrm{C})$.

\section{Funding information}

Funding for this research was provided by: Air Force Office of Scientific Research; National Research Council (award to LCB).

\section{References}

Cai, F., Li, L., Zhu, C., Li, J., Peng, H. \& Zou, Y. (2019). Chem. Phys. Lett. 730, 271-276.

Charpe, V. P., Sagadevan, A. \& Hwang, K. C. (2020). Green Chem. 22, 4426-4432.

Dolomanov, O. V., Bourhis, L. J., Gildea, R. J., Howard, J. A. K. \& Puschmann, H. (2009). J. Appl. Cryst. 42, 339-341.

El Moncef, A., Cuquerella, M. C., Zaballos, E., Ramírez de Arellano, C., Ben-Tama, A., Stiriba, S. E. \& Pérez-Prieto, J. (2010). Chem. Commun. 46, 800-802.

Fun, H.-K. \& Kia, R. (2008). Acta Cryst. E64, o1617.
Table 2

Experimental details.

\begin{tabular}{|c|c|}
\hline \multicolumn{2}{|l|}{ Crystal data } \\
\hline Chemical formula & $\mathrm{C}_{14} \mathrm{H}_{6} \mathrm{~F}_{4} \mathrm{O}_{2}$ \\
\hline$M_{\mathrm{r}}$ & 282.19 \\
\hline Crystal system, space group & Orthorhombic, $P b c n$ \\
\hline Temperature (K) & 100 \\
\hline$a, b, c(\AA)$ & $7.0588(2), 7.4008(2), 21.5265(4)$ \\
\hline$V\left(\AA^{3}\right)$ & $1124.56(5)$ \\
\hline$Z$ & 4 \\
\hline Radiation type & Mo $K \alpha$ \\
\hline$\mu\left(\mathrm{mm}^{-1}\right)$ & 0.16 \\
\hline Crystal size $(\mathrm{mm})$ & $0.30 \times 0.14 \times 0.10$ \\
\hline \multicolumn{2}{|l|}{ Data collection } \\
\hline Diffractometer & $\begin{array}{l}\text { XtaLAB Synergy, Single source at } \\
\text { offset/far, HyPix } 3000\end{array}$ \\
\hline Absorption correction & $\begin{array}{l}\text { Gaussian (CrysAlis PRO; Rigaku } \\
\quad \text { OD, 2020) }\end{array}$ \\
\hline$T_{\min }, T_{\max }$ & $0.679,1.000$ \\
\hline $\begin{array}{l}\text { No. of measured, independent and } \\
\text { observed }[I>2 \sigma(I)] \text { reflections }\end{array}$ & $13347,1202,1014$ \\
\hline$R_{\text {int }}$ & 0.034 \\
\hline$(\sin \theta / \lambda)_{\max }\left(\AA^{-1}\right)$ & 0.641 \\
\hline \multicolumn{2}{|l|}{ Refinement } \\
\hline$R\left[F^{2}>2 \sigma\left(F^{2}\right)\right], w R\left(F^{2}\right), S$ & $0.032,0.085,1.03$ \\
\hline No. of reflections & 1202 \\
\hline No. of parameters & 91 \\
\hline $\mathrm{H}$-atom treatment & H-atom parameters constrained \\
\hline$\Delta \rho_{\max }, \Delta \rho_{\min }\left(\mathrm{e} \AA^{-3}\right)$ & $0.21,-0.24$ \\
\hline
\end{tabular}

Computer programs: CrysAlis PRO (Rigaku OD, 2020), SHELXT (Sheldrick, 2015a), SHELXL (Sheldrick, 2015b), and OLEX2 (Dolomanov et al., 2009).

Goossens, D. J., Welberry, T. R., Heerdegen, A. P. \& Edwards, A. J. (2005). Z. Kristallogr. 220, 1035-1042.

Groom, C. R., Bruno, I. J., Lightfoot, M. P. \& Ward, S. C. (2016). Acta Cryst. B72, 171-179.

Jones, D. J., Purushothaman, B., Ji, S., Holmes, A. B. \& Wong, W. W. H. (2012). Chem. Commun. 48, 8066-8068.

Liu, J., Xu, L., Bai, J., Du, A. \& Wu, B. (2019). New J. Chem. 43, 82908298.

Rigaku OD (2020). CrysAlis PRO, CrysAlis CCD and CrysAlis RED. Rigaku Oxford Diffraction, Yarnton, England.

Sheldrick, G. M. (2015a). Acta Cryst. A71, 3-8.

Sheldrick, G. M. (2015b). Acta Cryst. C71, 3-8. 


\section{supporting information}

Acta Cryst. (2021). E77, 700-702 [https://doi.org/10.1107/S2056989021005363]

\section{Crystal structure of 1,2-bis(3,5-difluorophenyl)ethane-1,2-dione}

\section{Loren C. Brown and Gary J. Balaich}

\section{Computing details}

Data collection: CrysAlis PRO (Rigaku OD, 2020); cell refinement: CrysAlis PRO (Rigaku OD, 2020); data reduction: CrysAlis PRO (Rigaku OD, 2020); program(s) used to solve structure: SHELXT (Sheldrick, 2015a); program(s) used to refine structure: SHELXL (Sheldrick, 2015b); molecular graphics: OLEX2 (Dolomanov et al., 2009); software used to prepare material for publication: OLEX2 (Dolomanov et al., 2009).

1,2-Bis(3,5-difluorophenyl)ethane-1,2-dione

Crystal data

$\mathrm{C}_{14} \mathrm{H}_{6} \mathrm{~F}_{4} \mathrm{O}_{2}$

$M_{r}=282.19$

Orthorhombic, $\mathrm{Pbcn}$

$a=7.0588(2) \AA$

$b=7.4008(2) \AA$

$c=21.5265$ (4) $\AA$

$V=1124.56(5) \AA^{3}$

$Z=4$

$F(000)=568$

\section{Data collection}

XtaLAB Synergy, Single source at offset/far, HyPix 3000 diffractometer

Radiation source: micro-focus sealed X-ray tube, PhotonJet (Mo) X-ray Source

Mirror monochromator

Detector resolution: 10.0000 pixels $\mathrm{mm}^{-1}$

$\omega$ scans

Absorption correction: gaussian

(CrysAlisPro; Rigaku OD, 2020)

Refinement

Refinement on $F^{2}$

Least-squares matrix: full

$R\left[F^{2}>2 \sigma\left(F^{2}\right)\right]=0.032$

$w R\left(F^{2}\right)=0.085$

$S=1.03$

1202 reflections

91 parameters

0 restraints

Primary atom site location: dual
$D_{\mathrm{x}}=1.667 \mathrm{Mg} \mathrm{m}^{-3}$

Mo $K \alpha$ radiation, $\lambda=0.71073 \AA$

Cell parameters from 7273 reflections

$\theta=1.9-27.0^{\circ}$

$\mu=0.16 \mathrm{~mm}^{-1}$

$T=100 \mathrm{~K}$

Block, colourless

$0.30 \times 0.14 \times 0.10 \mathrm{~mm}$

$T_{\min }=0.679, T_{\max }=1.000$

13347 measured reflections

1202 independent reflections

1014 reflections with $I>2 \sigma(I)$

$R_{\text {int }}=0.034$

$\theta_{\max }=27.1^{\circ}, \theta_{\min }=1.9^{\circ}$

$h=-8 \rightarrow 9$

$k=-9 \rightarrow 9$

$l=-27 \rightarrow 26$

Hydrogen site location: inferred from neighbouring sites

$\mathrm{H}$-atom parameters constrained

$w=1 /\left[\sigma^{2}\left(F_{\mathrm{o}}^{2}\right)+(0.0435 P)^{2}+0.4818 P\right]$

where $P=\left(F_{\mathrm{o}}^{2}+2 F_{\mathrm{c}}^{2}\right) / 3$

$(\Delta / \sigma)_{\max }<0.001$

$\Delta \rho_{\max }=0.21 \mathrm{e} \AA^{-3}$

$\Delta \rho_{\min }=-0.24 \mathrm{e} \AA^{-3}$ 


\section{Special details}

Geometry. All esds (except the esd in the dihedral angle between two 1.s. planes) are estimated using the full covariance matrix. The cell esds are taken into account individually in the estimation of esds in distances, angles and torsion angles; correlations between esds in cell parameters are only used when they are defined by crystal symmetry. An approximate (isotropic) treatment of cell esds is used for estimating esds involving l.s. planes.

Fractional atomic coordinates and isotropic or equivalent isotropic displacement parameters $\left(\AA^{2}\right)$

\begin{tabular}{lllll}
\hline & $x$ & $y$ & $z$ & $U_{\text {iso }} * U_{\text {eq }}$ \\
\hline F1 & $1.04484(11)$ & $0.64342(12)$ & $0.45793(3)$ & $0.0274(2)$ \\
F2 & $0.41188(11)$ & $0.52722(13)$ & $0.40758(4)$ & $0.0298(2)$ \\
O1 & $0.78193(13)$ & $0.28658(13)$ & $0.22568(4)$ & $0.0236(3)$ \\
C1 & $0.97690(19)$ & $0.50922(17)$ & $0.36170(6)$ & $0.0183(3)$ \\
H1 & 1.1050 & 0.5083 & 0.3516 & $0.022^{*}$ \\
C2 & $0.91452(19)$ & $0.57731(18)$ & $0.41774(6)$ & $0.0196(3)$ \\
C3 & $0.72718(19)$ & $0.58412(18)$ & $0.43505(6)$ & $0.0200(3)$ \\
H3 & 0.6896 & 0.6293 & 0.4734 & $0.024^{*}$ \\
C4 & $0.59783(19)$ & $0.51991(19)$ & $0.39216(6)$ & $0.0200(3)$ \\
C5 & $0.64773(18)$ & $0.44830(18)$ & $0.33558(6)$ & $0.0190(3)$ \\
H5 & 0.5563 & 0.4056 & 0.3081 & $0.023^{*}$ \\
C6 & $0.84090(18)$ & $0.44170(17)$ & $0.32055(6)$ & $0.0167(3)$ \\
C7 & $0.89509(18)$ & $0.36177(17)$ & $0.25965(6)$ & $0.0174(3)$
\end{tabular}

Atomic displacement parameters $\left(\AA^{2}\right)$

\begin{tabular}{lllllll}
\hline & $U^{11}$ & $U^{22}$ & $U^{33}$ & $U^{12}$ & $U^{13}$ & $U^{23}$ \\
\hline F1 & $0.0235(5)$ & $0.0368(5)$ & $0.0218(4)$ & $-0.0053(4)$ & $-0.0033(3)$ & $-0.0094(3)$ \\
F2 & $0.0151(4)$ & $0.0487(6)$ & $0.0254(5)$ & $0.0037(4)$ & $0.0047(3)$ & $-0.0003(4)$ \\
O1 & $0.0235(5)$ & $0.0298(6)$ & $0.0176(5)$ & $-0.0056(4)$ & $-0.0014(4)$ & $-0.0014(4)$ \\
C1 & $0.0160(6)$ & $0.0201(7)$ & $0.0188(7)$ & $-0.0005(5)$ & $0.0007(5)$ & $0.0009(5)$ \\
C2 & $0.0203(7)$ & $0.0210(7)$ & $0.0174(6)$ & $-0.0013(5)$ & $-0.0030(5)$ & $-0.0009(5)$ \\
C3 & $0.0232(7)$ & $0.0200(7)$ & $0.0168(6)$ & $0.0026(6)$ & $0.0025(5)$ & $-0.0003(5)$ \\
C4 & $0.0142(7)$ & $0.0240(7)$ & $0.0220(7)$ & $0.0024(5)$ & $0.0028(5)$ & $0.0046(5)$ \\
C5 & $0.0170(6)$ & $0.0230(7)$ & $0.0168(6)$ & $-0.0012(5)$ & $-0.0023(5)$ & $0.0029(5)$ \\
C6 & $0.0169(7)$ & $0.0168(7)$ & $0.0162(6)$ & $0.0000(5)$ & $0.0005(5)$ & $0.0024(5)$ \\
C7 & $0.0184(7)$ & $0.0178(6)$ & $0.0161(6)$ & $-0.0001(5)$ & $-0.0011(5)$ & $0.0036(5)$ \\
\hline
\end{tabular}

Geometric parameters $\left(\AA,{ }^{\circ}\right)$

\begin{tabular}{llll}
\hline $\mathrm{F} 1-\mathrm{C} 2$ & $1.3543(15)$ & $\mathrm{C} 3-\mathrm{H} 3$ & 0.9300 \\
$\mathrm{~F} 2-\mathrm{C} 4$ & $1.3550(15)$ & $\mathrm{C} 3-\mathrm{C} 4$ & $1.3827(19)$ \\
$\mathrm{O} 1-\mathrm{C} 7$ & $1.2176(16)$ & $\mathrm{C} 4-\mathrm{C} 5$ & $1.3741(19)$ \\
$\mathrm{C} 1-\mathrm{H} 1$ & 0.9300 & $\mathrm{C} 5-\mathrm{H} 5$ & 0.9300 \\
$\mathrm{C} 1-\mathrm{C} 2$ & $1.3796(18)$ & $\mathrm{C} 5-\mathrm{C} 6$ & $1.4023(18)$ \\
$\mathrm{C} 1-\mathrm{C} 6$ & $1.3985(18)$ & $\mathrm{C} 6-\mathrm{C} 7$ & $1.4882(17)$ \\
$\mathrm{C} 2-\mathrm{C} 3$ & $1.3748(19)$ & $\mathrm{C} 7-\mathrm{C} 7^{\mathrm{i}}$ & $1.538(3)$ \\
$\mathrm{C} 2-\mathrm{C} 1-\mathrm{H} 1$ & 121.1 & $\mathrm{C} 5-\mathrm{C} 4-\mathrm{C} 3$ & $123.72(13)$
\end{tabular}




$\begin{array}{llll}\mathrm{C} 2-\mathrm{C} 1-\mathrm{C} 6 & 117.73(12) & \mathrm{C} 4-\mathrm{C} 5-\mathrm{H} 5 & 121.1 \\ \mathrm{C} 6-\mathrm{C} 1-\mathrm{H} 1 & 121.1 & \mathrm{C} 4-\mathrm{C} 5-\mathrm{C} 6 & 117.85(12) \\ \mathrm{F} 1-\mathrm{C} 2-\mathrm{C} 1 & 118.28(11) & \mathrm{C} 6-\mathrm{C} 5-\mathrm{H} 5 & 121.1 \\ \mathrm{~F} 1-\mathrm{C} 2-\mathrm{C} 3 & 117.84(11) & \mathrm{C} 1-\mathrm{C} 6-\mathrm{C} 5 & 120.59(12) \\ \mathrm{C} 3-\mathrm{C} 2-\mathrm{C} 1 & 123.87(13) & \mathrm{C} 1-\mathrm{C} 6-\mathrm{C} 7 & 121.56(12) \\ \mathrm{C} 2-\mathrm{C} 3-\mathrm{H} 3 & 121.9 & \mathrm{C} 5-\mathrm{C} 6-\mathrm{C} 7 & 117.84(11) \\ \mathrm{C} 2-\mathrm{C} 3-\mathrm{C} 4 & 116.21(12) & \mathrm{O} 1-\mathrm{C} 7-\mathrm{C} 6 & 122.83(12) \\ \mathrm{C} 4-\mathrm{C} 3-\mathrm{H} 3 & 121.9 & \mathrm{O} 1-\mathrm{C} 7-\mathrm{C} 7^{\mathrm{i}} & 117.99(12) \\ \mathrm{F} 2-\mathrm{C} 4-\mathrm{C} 3 & 117.54(12) & \mathrm{C} 6-\mathrm{C} 7-\mathrm{C} 7^{\mathrm{i}} & 119.05(12) \\ \mathrm{F} 2-\mathrm{C} 4-\mathrm{C} 5 & 118.74(12) & & \end{array}$

Symmetry code: (i) $-x+2, y,-z+1 / 2$.

Hydrogen-bond geometry $\left(\AA,{ }^{\circ}\right)$

\begin{tabular}{lllll}
\hline$D-\mathrm{H} \cdots A$ & $D-\mathrm{H}$ & $\mathrm{H} \cdots A$ & $D \cdots A$ & $D-\mathrm{H}^{\cdots} A$ \\
\hline $\mathrm{C} 1-\mathrm{H} 1 \cdots \mathrm{F} 2^{\mathrm{ii}}$ & 0.93 & 2.48 & $3.2281(16)$ & 137 \\
$\mathrm{C} 3-\mathrm{H} 3 \cdots \mathrm{F} 1^{\mathrm{iii}}$ & 0.93 & 2.46 & $3.3211(15)$ & 154 \\
$\mathrm{C} 5-\mathrm{H} 5 \cdots \mathrm{O} 1^{\text {iv }}$ & 0.93 & 2.65 & $3.517(2)$ & 156 \\
\hline
\end{tabular}

Symmetry codes: (ii) $x+1, y, z$; (iii) $x-1 / 2,-y+3 / 2,-z+1$; (iv) $-x+1, y,-z+1 / 2$.

Selected bond distances $(A)$ and angles $\left(^{\circ}\right)$ for 1,2-bis(3,5-difluorophenyl)ethane-1,2-dione.

\begin{tabular}{ll}
\hline $\mathrm{C} 1-\mathrm{C} 2$ & $1.3796(18)$ \\
$\mathrm{C} 1-\mathrm{C} 6$ & $1.3985(18)$ \\
$\mathrm{C} 2-\mathrm{C} 3$ & $1.3748(19)$ \\
$\mathrm{C} 3-\mathrm{C} 4$ & $1.3827(19)$ \\
$\mathrm{C} 4-\mathrm{C} 5$ & $1.3741(19)$ \\
$\mathrm{C} 5-\mathrm{C} 6$ & $1.4023(18)$ \\
$\mathrm{C} 6-\mathrm{C} 7$ & $1.4882(17)$ \\
$\mathrm{C} 7-\mathrm{C} 7$ & $1.538(3)$ \\
$\mathrm{C} 7-\mathrm{O} 1$ & $1.2176(16)$ \\
$\mathrm{F} 1-\mathrm{C} 2$ & $1.3543(15)$ \\
$\mathrm{F} 2-\mathrm{C} 4$ & $1.3550(15)$ \\
& \\
$\mathrm{C} 1-\mathrm{C} 6-\mathrm{C} 5$ & $120.59(12)$ \\
$\mathrm{C} 1-\mathrm{C} 6-\mathrm{C} 7$ & $121.56(12)$ \\
$\mathrm{C} 2-\mathrm{C} 1-\mathrm{C} 6$ & $117.73(12)$ \\
$\mathrm{C} 2-\mathrm{C} 3-\mathrm{C} 4$ & $116.21(12)$ \\
$\mathrm{C} 3-\mathrm{C} 2-\mathrm{C} 1$ & $123.87(13)$ \\
$\mathrm{C} 4-\mathrm{C} 5-\mathrm{C} 6$ & $117.85(12)$ \\
$\mathrm{C} 5-\mathrm{C} 4-\mathrm{C} 3$ & $123.72(13)$ \\
$\mathrm{C} 5-\mathrm{C} 6-\mathrm{C} 7$ & $117.84(11)$ \\
$\mathrm{C} 6-\mathrm{C} 7-\mathrm{C} 7{ }^{\prime}$ & $119.05(12)$ \\
$\mathrm{O} 1-\mathrm{C} 7-\mathrm{C} 6$ & $122.83(12)$ \\
$\mathrm{O} 1-\mathrm{C} 7-\mathrm{C} 7^{\prime}$ & $117.99(12)$ \\
$\mathrm{F} 1-\mathrm{C} 2-\mathrm{C} 1$ & $118.28(11)$ \\
$\mathrm{F} 1-\mathrm{C} 2-\mathrm{C} 3$ & $117.84(11)$
\end{tabular}


F2-C4-C3

$117.54(12)$

F2-C4-C5

$118.74(12)$ 